\title{
Glucagon like peptide-1 receptor agonists for the management of obesity and non-alcoholic fatty liver disease: a novel therapeutic option
}

\author{
Gauri Dhir, ${ }^{1,2}$ Kenneth Cusi ${ }^{1,2}$
}

${ }^{1}$ Division of Endocrinology, Diabetes and Metabolism, University of Florida, Gainesville, Florida, USA ${ }^{2}$ Division of Endocrinology, Diabetes and Metabolism, Malcom Randall VA Medical Center, Gainesville, Florida, USA

\section{Correspondence to} Dr Kenneth Cusi, Department of Endocrinology, Diabetes and Metabolism Division University of Florida, Gainesville, Florida 32610, USA; kcusi@ufl.edu

Accepted 12 August 2017 Published Online First 15 September 2017
CrossMark

\author{
To cite: Dhir G, \\ Cusi K. J Investig Med \\ 2018;66:7-10.
}

\begin{abstract}
Obesity is a major risk factor for the development of type 2 diabetes mellitus (T2DM), and is associated with a cluster of metabolic factors that lead to poor cardiovascular outcomes. In non-alcoholic fatty liver disease (NAFLD), liver fat (triglyceride) accumulation closely mirrors adipose tissue dysfunction and insulin resistance in obesity and T2DM. It is now recognized as the most common chronic liver disease in Westernized societies, often progressing to more severe forms of the disease such as nonalcoholic steatohepatitis (NASH), or cirrhosis and hepatocellular carcinoma. However, NAFLD remains largely overlooked by healthcare providers although it affects about two-thirds of patients with obesity and it promotes the development of T2DM. NAFLD mirrors adipose tissue and systemic insulin resistance, the liver being a 'barometer' of metabolic health. Although pioglitazone is emerging as the treatment of choice for NASH in patients with insulin-resistance, or those with T2DM, many other options are being tested. Due to their overall safety and efficacy, glucagon-like peptide-1 receptor agonists (GLP-1 RAs) are becoming one of the cornerstones for the management of both obesity and T2DM, and a novel alternative for the treatment of NAFLD. In this review, we will briefly summarize the status of GLP-1RA for the treatment of obesity and NAFLD.
\end{abstract}

Obesity is a worldwide problem affecting over a third of the US adult population. ${ }^{1}$ It is regarded as the epidemic of the 21st century with global estimates of about 500 million people having obesity and at risk of significant obesity-related morbidity. ${ }^{2}$ NAFLD describes a spectrum of liver diseases ranging from simple steatosis, to its more severe form known as nonalcoholic steatohepatitis (NASH) with hepatocyte injury, liver inflammation, often with fibrosis. Patients usually are obese or have type 2 diabetes mellitus (T2DM) and are at an increased risk of developing cirrhosis and hepatocellular carcinoma. ${ }^{3}$ It is believed that NAFLD will become the leading cause of liver transplantation by 2020 in parallel with the increase in the obesity epidemic. ${ }^{3-6}$ About $70 \%$ or more of obese individuals (and especially those with T2DM) have NAFLD and as many as 50\% of them may have NASH. ${ }^{135}$ Development of NASH is also associated with a higher risk of death from cardiovascular disease (CVD).$^{3-5}$

\section{TREATMENT OF OBESITY AND NAFLD}

Lifestyle intervention is the mainstay of the treatment for obesity. However, weight regain is very common and lifestyle intervention is often ineffective in the maintenance of long-term weight loss. ${ }^{2}$ There are currently five Food and Drug Administration (FDA) approved drugs for obesity in the USA: lorcaserin (Belviq), orilstat (Xenical), phentermine-topiramate (Qsymia), bupropion-naltrexone (Contrave) and liraglutide (Saxenda). ${ }^{1278}$ Most of the antiobesity drugs have faced obstacles in their acceptance by primary care providers due to their high cost, limited efficacy and/or side effects. In addition, there is a lack of controlled studies on their long-term health benefits. ${ }^{8}$

Lifestyle intervention has also proven to be effective in the management of NAFLD. In shortterm studies (most 6-12 months), reduction of $\sim 10 \%$ in body weight results in a significant improvement in necroinflammation and often of fibrosis in NASH. ${ }^{3-5}$ However, it is difficult to maintain long-term weight loss with dietary modification alone, and many therapeutic options have been studied for the treatment of NASH, although none are FDA-approved. Pioglitazone (Actos) has emerged as the treatment of choice for patients with and without T2DM, improving the metabolic profile and inducing resolution of NASH in $47 \%-58 \%$ of the patients. ${ }^{3-5}$ From the first proof-of-concept randomized controlled trial (RCT) in patients with prediabetes or T2DM and $\mathrm{NASH}^{9}$ to a recent larger, 3-year long-term study in this same population, pioglitazone has been found to be safe and to significantly improve liver histology in about two-thirds of patients with NASH. ${ }^{10}$ Vitamin $\mathrm{E}$ has been used with some success in non-diabetic patients. ${ }^{3}$ Newer agents tested including obeticholic acid and elafibranor have shown modest improvement on liver histology in NASH, but larger trials fully examining their safety and efficacy are ongoing. ${ }^{5}$ However, glucagon-like peptide-1 receptor agonists (GLP-1 RAs) have recently emerged as an attractive treatment option for patients with obesity and NASH. 


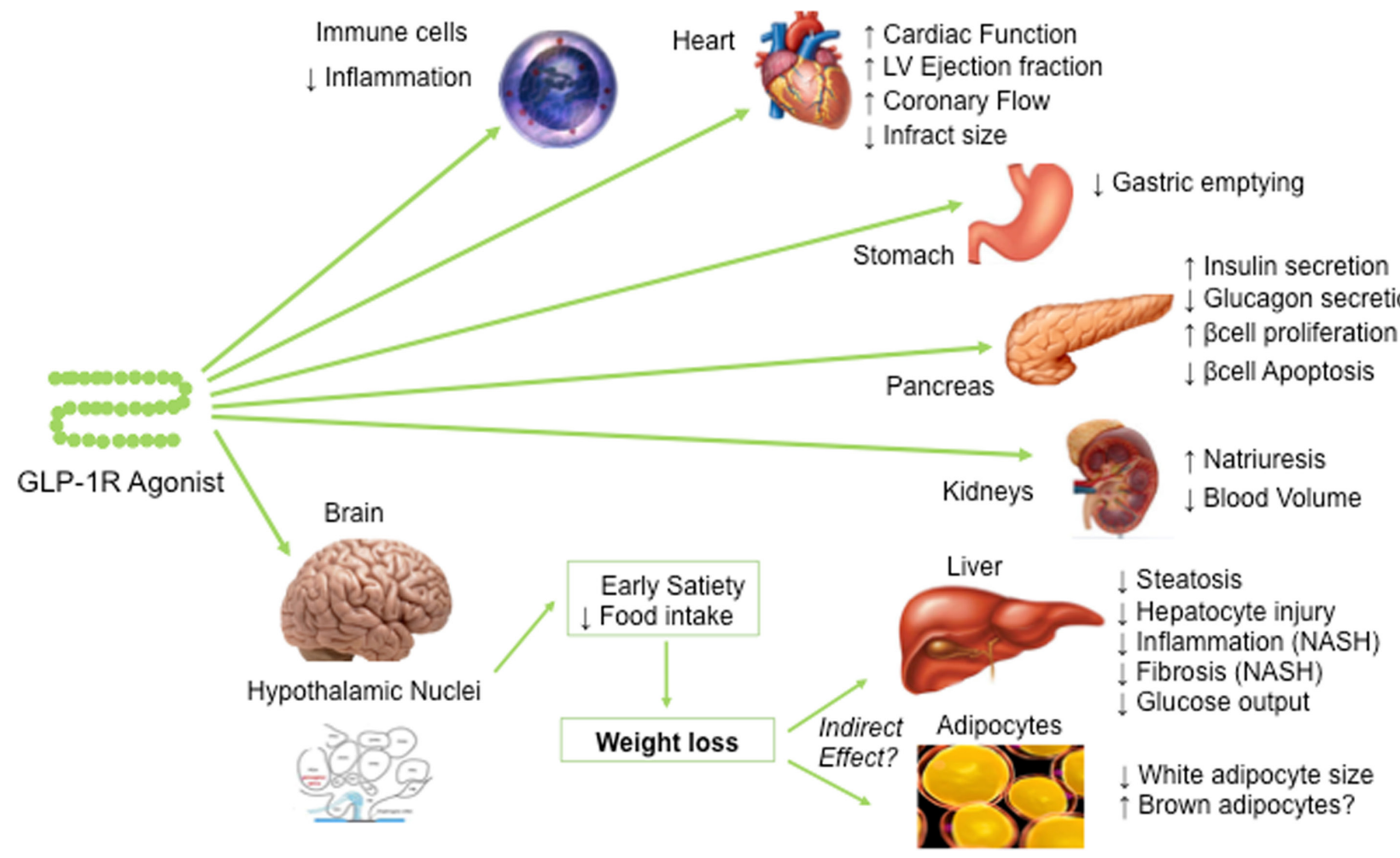

Figure 1 Physiological effects of glucagon-like peptide-1 receptor agonists (GLP-1RAs) in humans.

\section{BIOLOGY OF GLP-1RA}

Among the several intestinal peptides secreted from gut by neuroendocrine cells, the native GLP-1 (7-36 and 7-37 amide) has been the most extensively studied and possibly the one with the greatest translational relevance. ${ }^{11} 12$ Figure 1 illustrates the broad spectrum of their metabolic effects including an increase in glucose-dependent insulin secretion, stimulation of $\beta$-cell proliferation, inhibition of glucagon secretion, increased satiety acting through central nervous system pathways and delayed gastric emptying. GLP-1 exerts direct effects through the class B family of G protein coupled receptors and exerts indirect non-receptor mediated effects. ${ }^{12}$ The glucagon-like peptide- 1 receptor (GLP-1R) is widely expressed in islet cells and extrapancreatic tissues like lung, atrial myocytes, kidney, gastrointestinal tract, peripheral and central nervous systems, vascular smooth muscle and lymphocytes. ${ }^{11}$ The expression of GLP-1R in hepatocytes is controversial. While some studies claim to have identified receptors in hepatocytes with effects on insulin sensitivity and some anti-inflammatory effects, ${ }^{13-15}$ others have failed to reach a similar conclusion and report no GLP-1R in the liver ${ }^{16}{ }^{17}$ (also reviewed in ref. 1,11$)$. While the direct effects of GLP-1 on adipose tissue remain to be fully understood, at the present time it is believed that most of the weight loss induced by GLP-1RAs is from effects on the central nervous system by induction of satiety, although there is some evidence that GLP-1 may increase thermogenesis in brown adipose tissue. ${ }^{11}$

\section{GLP-1RA FOR THE TREATMENT OF OBESITY}

The most extensively studied GLP-1RA for the treatment of obesity is liraglutide (as Saxenda in a $3 \mathrm{mg}$ per day formulation). It was approved specifically by the FDA for the management of obesity based on three 56-week RCTs (SCALE trials: Satiety and Clinical Adiposity-Liraglutide Evidence): SCALE Obesity ${ }^{18}$ SCALE Maintain, ${ }^{19}$ and SCALE Diabetes, ${ }^{20}$ examined the efficacy and safety of 3 mg of liraglutide in overweight or obese patients with or without T2DM. Taken together, liraglutide significantly reduced body weight compared with placebo by $\geq 5 \%$ in $\sim 30 \%$ of patients and by $\geq 10 \%$ in $\sim 15 \%$ of the participants. ${ }^{18-20}$ In a 3 -year extension in patients with obesity and prediabetes, $30 \%$ of the subjects reverted from prediabetes to normoglycemia with liraglutide. ${ }^{21}$ Other GLP-1RAs used for the treatment of T2DM have also had modest weight loss effects. ${ }^{12}$ However, albiglutide is not associated with significant weight loss and is not under investigation for the treatment in obese non-diabetic subjects. The novel GLP-1RA semaglutide in the phase-III (SUSTAIN-1) trial in patients with T2DM showed dose-dependent reduction in body weight up to $4.6 \mathrm{~kg}$ compared with $1 \mathrm{~kg}$ with placebo. ${ }^{12}$ An oral formulation of semaglutide is also under evaluation.

Recent clinical trials, such as the Liraglutide Effect and Action in Diabetes: Evaluation of Cardiovascular Outcome Results (LEADER) ${ }^{22}$ and SUSTAIN 6 (semaglutide and cardiovascular outcomes) ${ }^{23}$ have demonstrated that GLP-1RAs can significantly reduce cardiovascular events in patients with T2DM. Because of this and its glucose-lowering efficacy, liraglutide has been favored over other pharmacological agents for the treatment of obesity, particularly in patients with T2DM. There are several ongoing trials with various GLP-1 RAs in patients with obesity with and without T2DM. 


\section{GLP-1RA FOR THE TREATMENT OF NAFLD}

Recent work has linked the development of hepatocyte triglyceride accumulation in obesity to the presence of dysfunctional, insulin-resistant adipose tissue. ${ }^{1}$ This has been recently confirmed in patients with NAFLD in whom intrahepatic triglyceride accumulation, as well as hepatic and muscle insulin sensitivity, correlated closely with the severity of adipose tissue insulin resistance. ${ }^{24} 25$ Within this context, although many pharmacological agents are undergoing active investigation, ${ }^{26}$ testing the efficacy of GLP-1RAs in NASH appeared only as a logical consequence of their beneficial metabolic effects in insulin-resistant states. ${ }^{1} 111227$

Early studies in patients with T2DM suggested the potential benefit of GLP- 1RA from a reduction in liver enzymes. ${ }^{27}$ This reduction in plasma aminotransferases was also associated with a reduction in intrahepatic triglycerides proportional to the degree of weight $\operatorname{loss}^{28-30}$ or improvement in glycemic control. ${ }^{4}$ Small early trials and case control studies (including less than 20 patients each) also suggested a benefit for exenatide ${ }^{31}$ or liraglutide ${ }^{32} 33$ on liver fat and histology. However, the LEAN trial (Liraglutide Efficacy and Action in NASH) ${ }^{6}$ was the first RCT that as a proof of concept $(n=52)$ reported on the true efficacy of a GLP-1RA in patients with biopsy-proven NASH. After 48 weeks of treatment with liraglutide (1.8 mg per day), resolution of NASH occurred in 9/23 (39\%) compared with $2 / 22(9 \%)$ participants in the placebo group. These results have created a new treatment paradigm in the field. This study is being followed up by a large multicenter trial testing three doses of semaglutide once daily versus placebo in patients with biopsy-proven NASH (ClinicalTrials.gov NCT02970942). Using the same formulation as being used in the above NASH trial, results from a recently released 52-week RCT in 957 patients with obesity at doses between $0.05 \mathrm{mg} /$ day to $0.4 \mathrm{mg} /$ day (or placebo) led to an estimated $13.8 \%$ weight loss versus $2.3 \%$ in patients at placebo alone (Novo Nordisk press release, June 23, 2017). Such results are highly promising for the treatment of NASH.

At least in part the success of GLP-1RAs is based on the fact that they are well tolerated, except for a modest increase in gastrointestinal symptoms of nausea and diarrhea. ${ }^{12}$ Current evidence does not support the hypothesis of an increased risk of pancreatitis. A recent meta-analysis of placebo-controlled GLP-1RA RCTs with a minimum duration of 24 months, including a total of 9347 GLP-1RAtreated and 9353 placebo-treated patients, in which acute pancreatitis was a predefined adverse event and adjudicated by blinded and independent adjudicating committees, found no evidence that treatment with GLP-1RA increases the risk in patients with T2DM. ${ }^{34}$ Monami $e t ~ a l^{35}$ reported from a review of 100 trials reporting on pancreatitis and pancreatic cancer, that the prevalence of these events in GLP1-RA was not significantly different from that observed in comparator arms (OR (95\% CI) of 0.93 (0.65 to 1.34), $\mathrm{p}=0.71$, and 0.94 (0.52 to 1.70$), p=0.84$, for pancreatitis and pancreatic cancer, respectively). Of note, they did report on a significantly increased risk of cholelithiasis (OR $(95 \% \mathrm{CI}) 1.30$ (1.01 to 1.68$), \mathrm{p}=0.041$ ) that deserves further exploration. In addition, there was no signal for pancreatic cancer in recent large RCTs. ${ }^{18-21}$

\section{CONCLUSION}

Treatment with GLP-1RA of patients with T2DM offers additional value beyond glycemic control as they aid in the management of obesity and reduce the high CVD risk associated with both conditions. Their unique ability to promote weight loss, improve glycemic control and potentially to reverse hepatocyte injury and liver inflammation and fibrosis, make GLP-1RAs a novel and attractive therapeutic option for the treatment of NASH. Larger long-term RCTs will likely help define their role in management of these complex patients in the near future.

Contributors GD performed the initial review of the available literature, created figure 1 and wrote the initial manuscript draft; KC reviewed the literature, mentored GD and worked on revising and submitting the final version of the manuscript.

Competing interests None declared.

Provenance and peer review Commissioned; externally peer reviewed.

(c) American Federation for Medical Research (unless otherwise stated in the text of the article) 2018. All rights reserved. No commercial use is permitted unless otherwise expressly granted.

\section{REFERENCES}

1 Cusi K. Role of obesity and lipotoxicity in the development of nonalcoholic steatohepatitis: pathophysiology and clinical implications. Gastroenterology 2012;142:711-25.

2 Garvey WT, Mechanick JI, Brett EM, et al. American association of clinical endocrinologists and american college of endocrinology comprehensive clinica practice guidelines for medical care of patients with obesity. Endocr Pract 2016;22(Suppl 3):1-203.

3 Bril F, Cusi K. Nonalcoholic fatty liver disease: the new complication of type 2 diabetes mellitus. Endocrinol Metab Clin North Am 2016;45:765-81.

4 Cuthbertson DJ, Irwin A, Gardner CJ, et al. Improved glycaemia correlates with liver fat reduction in obese, type 2 diabetes, patients given glucagon-like peptide-1 (GLP-1) receptor agonists. PLoS One 2012;7:e50117.

5 Bril F, Cusi K. Management of nonalcoholic fatty liver disease in patients with type 2 diabetes: a call to action. Diabetes Care 2017;40:419-30.

6 Armstrong MJ, Gaunt P, Aithal GP, et al. Liraglutide safety and efficacy in patients with non-alcoholic steatohepatitis (LEAN): a multicentre, double-blind, randomised, placebo-controlled phase 2 study. Lancet 2016;387:679-90.

7 Isaacs D, Prasad-Reddy L, Srivastava SB. Role of glucagon-like peptide 1 receptor agonists in management of obesity. Am J Health Syst Pharm 2016;73:1493-507.

8 Saunders $\mathrm{KH}$, Kumar RB, Igel LI, et al. Pharmacologic approaches to weight management: recent gains and shortfalls in combating obesity. Curr Atheroscler Rep 2016:18:36.

9 Belfort R, Harrison SA, Brown K, et al. A placebo-controlled trial of pioglitazone in subjects with nonalcoholic steatohepatitis. N Engl J Med 2006;355:2297-307.

10 Cusi K, Orsak B, Bril F, et al. Long-term pioglitazone treatment for patients with nonalcoholic steatohepatitis and prediabetes or type 2 diabetes mellitus: a randomized trial. Ann Intern Med 2016;165:305-15.

11 Drucker DJ. Deciphering metabolic messages from the gut drives therapeutic innovation: the 2014 Banting Lecture. Diabetes 2015;64:317-26.

12 Nauck M. Incretin therapies: highlighting common features and differences in the modes of action of glucagon-like peptide-1 receptor agonists and dipeptidyl peptidase-4 inhibitors. Diabetes Obes Metab 2016;18:203-16.

13 Gupta NA, Mells J, Dunham RM, et al. Glucagon-like peptide-1 receptor is present on human hepatocytes and has a direct role in decreasing hepatic steatosis in vitro by modulating elements of the insulin signaling pathway. Hepatology 2010;51:1584-92.

14 Rahman K, Liu Y, Kumar P, et al. C/EBP homologous protein modulates liraglutide-mediated attenuation of non-alcoholic steatohepatitis. Lab Invest 2016;96:895-908.

15 Zheng X, Xu F, Liang H, et al. SIRT1/HSF1/HSP pathway is essential for exenatide-alleviated, lipid-induced hepatic endoplasmic reticulum stress. Hepatology 2017;66:809-24.

16 Panjwani N, Mulvihill EE, Longuet C, et al. GLP-1 receptor activation indirectly reduces hepatic lipid accumulation but does not attenuate development of atherosclerosis in diabetic male $\mathrm{ApoE}(-/-)$ mice. Endocrinology 2013;154:127-39. 
17 Pyke C, Heller RS, Kirk RK, et al. GLP-1 receptor localization in monkey and human tissue: novel distribution revealed with extensively validated monoclonal antibody. Endocrinology 2014;155:1280-90.

18 Pi-Sunyer X, Astrup A, Fujioka K, et al. A randomized, controlled trial of $3.0 \mathrm{mg}$ of liraglutide in weight management. N Eng/ J Med 2015;373:11-22.

19 Wadden TA, Hollander P, Klein S, et al. Weight maintenance and additional weight loss with liraglutide after low-calorie-diet-induced weight loss: the SCALE Maintenance randomized study. Int J Obes 2013;37:1443-51.

20 Davies MJ, Bergenstal R, Bode B, et al. Efficacy of liraglutide for weight loss among patients with type 2 diabetes: the SCALE diabetes randomized clinical trial. JAMA 2015;314:687-99.

21 le Roux CW, Astrup A, Fujioka K, et al. 3 years of liraglutide versus placebo for type 2 diabetes risk reduction and weight management in individuals with prediabetes: a randomised, double-blind trial. Lancet 2017;389:1399-409.

22 Marso SP, Daniels GH, Brown-Frandsen K, et al. Liraglutide and cardiovascular outcomes in type 2 diabetes. N Eng/ J Med 2016;375:311-22.

23 Marso SP, Bain SC, Consoli A, et al. Semaglutide and cardiovascular outcomes in patients with type 2 diabetes. N Eng/ J Med 2016;375:1834-44.

24 Lomonaco R, Ortiz-Lopez C, Orsak B, et al. Effect of adipose tissue insulin resistance on metabolic parameters and liver histology in obese patients with nonalcoholic fatty liver disease. Hepatology 2012;55:1389-97.

25 Bril F, Barb D, Portillo-Sanchez P, et al. Metabolic and histological implications of intrahepatic triglyceride content in nonalcoholic fatty liver disease. Hepatology 2017;65:1132-44.

26 Cusi K. Treatment of patients with type 2 diabetes and non-alcoholic fatty liver disease: current approaches and future directions. Diabetologia 2016:59:1112-20.
27 Barb D, Portillo-Sanchez P, Cusi K. Pharmacological management of nonalcoholic fatty liver disease. Metabolism 2016;65:1183-95.

28 Ohki T, Isogawa A, Iwamoto $M$, et al. The effectiveness of liraglutide in nonalcoholic fatty liver disease patients with type 2 diabetes mellitus compared to sitagliptin and pioglitazone. Scientific World Journal 2012;2012:1-8.

29 Armstrong MJ, Houlihan DD, Rowe IA, et al. Safety and efficacy of liraglutide in patients with type 2 diabetes and elevated liver enzymes: individual patient data meta-analysis of the LEAD program. Aliment Pharmacol Ther 2013;37:234-42.

30 Petit JM, Cercueil JP, Loffroy R, et al. Effect of liraglutide therapy on liver fat content in patients with inadequately controlled type 2 diabetes: the LiraNAFLD study. J Clin Endocrinol Metab 2017;102:407-15.

31 Dong Y, Lv Q, Li S, et al. Efficacy and safety of glucagon-like peptide-1 receptor agonists in non-alcoholic fatty liver disease: A systematic review and metaanalysis. Clin Res Hepatol Gastroenterol 2017;41:284-95.

32 Eguchi Y, Kitajima Y, Hyogo H, et al. Pilot study of liraglutide effects in nonalcoholic steatohepatitis and non-alcoholic fatty liver disease with glucose intolerance in Japanese patients (LEAN-J). Hepatol Res 2015;45:269-78.

33 Jendle J, Nauck MA, Matthews DR, et al. Weight loss with liraglutide, a oncedaily human glucagon-like peptide- 1 analogue for type 2 diabetes treatment as monotherapy or added to metformin, is primarily as a result of a reduction in fat tissue. Diabetes Obes Metab 2009;11:1163-72.

34 Storgaard H, Cold F, Gluud LL, et al. Glucagon-like peptide-1 receptor agonists and risk of acute pancreatitis in patients with type 2 diabetes. Diabetes Obes Metab 2017;19:906-8.

35 Monami M, Nreu B, Scatena A, et al. Safety issues with glucagon-like peptide-1 receptor agonists (pancreatitis, pancreatic cancer and cholelithiasis): Data from randomized controlled trials. Diabetes Obes Metab 2017;19:1233-41. 